Modell Baden-Württemberg

\section{Das modulare Rahmenkonzept}

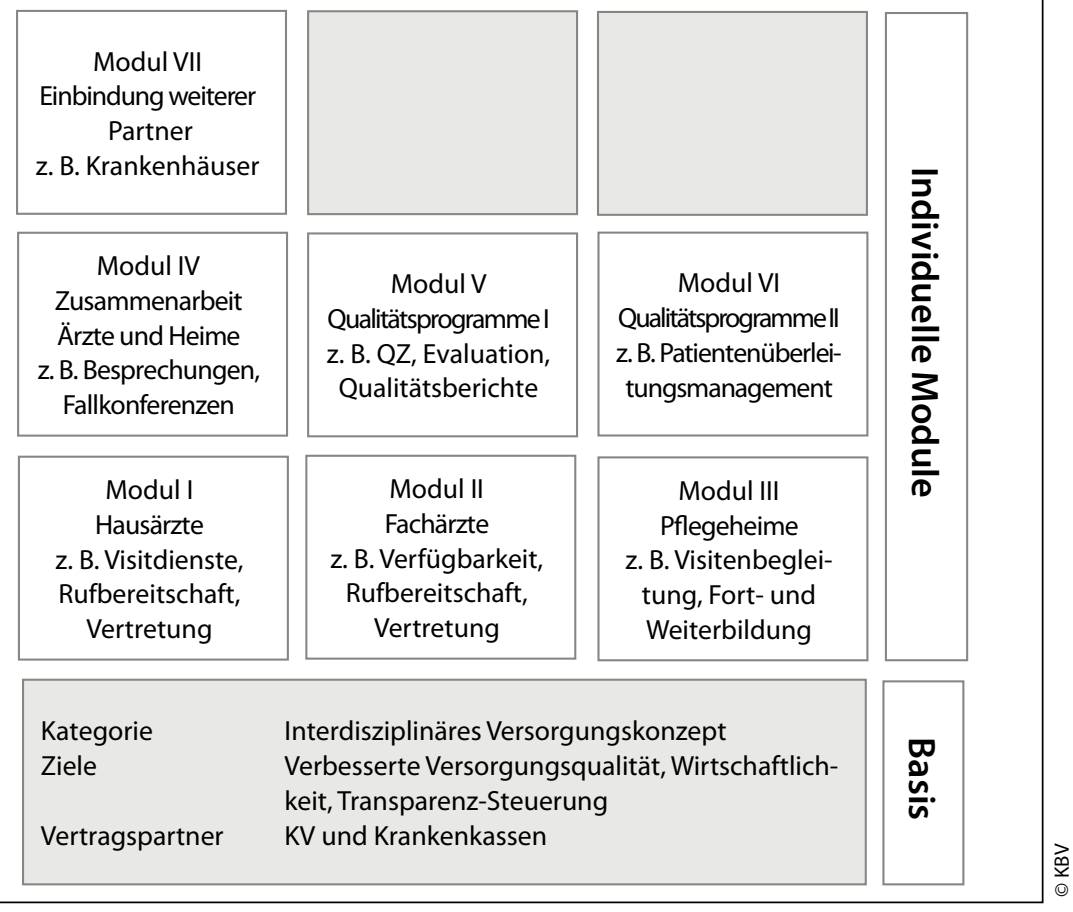

um 30\% angehoben und zusammen mit dem dringenden Heimbesuch nach Nr. 01415 EBM außerhalb der Regelleistungsvolumina vergütet. Ferner wurde im RLV-Beschluss zu den Praxisbesonderheiten (Ab- schnitt l., Nr. 3.7) aufgenommen, dass sich aus der ärztlichen Besuchstätigkeit in beschützenden Wohnheimen bzw. Einrichtungen sowie in Alten- oder Pflegeheimen ein besonderer Versorgungsauftrag ergeben kann, welcher eine Erhöhung des RLV oder betroffener QZV zur Berücksichtigung des erhöhten Leistungsbedarfs während der Besuche rechtfertigen kann.

Das klingt, als ob die Neuregelung in BadenWürttemberg zur zweimaligen Weihnachtsfeier führt. Tatsächlich stellen nur diese Sonderverträge eine Problemlösung dar, während die Hausärzte außerhalb Bayerns und Baden-Württembergs das Mehrhonorar durch Abstriche an anderer Stelle selbst bezahlen und allenfalls näher an eine Plausibilitätsprüfung heranrücken. Obgleich sich nämlich der Zeitaufwand bei einem Hausbesuch durch die Neubewertung nicht geändert haben kann, wurden die (Plausibilitäts-)Prüfzeiten bei der Nr. 01410 EBM von 15 auf 20 Minuten und bei der Nr. 01413 EBM von 5 auf 7 Minuten erhöht. Wer also bisher vier Patienten in einer Stunde besucht hat, darf jetzt nur noch drei Patienten in dieser Zeiteinheit behandeln.

Die Beschlüsse des Bewertungsausschusses führen dazu, dass der Mehrbedarf an Hausund Heimbesuchen einfach mit den Honorarsteigerungen 2011 abgedeckt wird. Die übrigen (Mehr-)Leistungen - insbesondere im RLV - müssen hingegen zum Nulltarif erbracht werden. Über einen solchen $A b$ schluss können allenfalls die Kassen jubeln!

\title{
Zieht die AKR-Karawane einfach ihren Weg?
}

- Die Front gegen die Ambulanten Kodierrichtlinien (AKR) wächst und wächst. Nach der Ärztekammer Hessen (LÄKH) hat nun auch die in Nordrhein (ÄKNo) ihre Mitglieder dazu aufgerufen, sich dem Protest anzuschließen und fordert, die Richtlinien erst nach einer breit angelegten Testphase einzuführen.

Zusammen mit den Kassenärztlichen Vereinigungen Hessen, Bayern und Niedersachsen ruft die Kammer darüber hinaus auf, die Petition eines baden-württembergischen Hausarztes an den Deutschen Bundestag zu unterstützen. Knapp
9000 Ärzte haben diese Petition „Ärzte Stopp der ambulanten Kodierrichtlinien" bereits mitgezeichnet. Damit sich die Parlamentarier des Themas annehmen, müssen bis zum 16. Februar 50000 Unterschriften zusammenkommen.

Die Kritik an den AKR konzentriert sich dabei immer mehr auf die Kassenärztliche Bundesvereinigung (KBV) als Verursacher. Zwar sind Kodierrichtlinien gesetzliche Pflicht und müssen befolgt werden. Die im Auftrag der KBV entwickelte Version der AKR stellt nach einhelliger Beurteilung aber eine weitere, unzumutbare bürokra- tische Belastung der Praxen dar, die einfach so nicht hingenommen werden darf.

\section{MMW Kommentar}

Fast scheint es, als ob die zunehmende Kritik an den AKR an der KBV spurlos vorübergeht. Im wöchentlichen Wechsel erscheint stattdessen im Deutschen Ärzteblatt ein Ratgeber zur Umsetzung der AKR. Auf dem CME-Portal des Internetauftrittes des Deutschen Ärzteblattes können Ärzte sogar insgesamt bis zu zehn CME-Punkte durch Teilnahme an dieser im Grunde genommen völlig sinnlosen Schulung erwerben. 